\title{
Minimal model for the fermion flavor structure, mass hierarchy, dark matter, leptogenesis, and the electron and muon anomalous magnetic moments
}

\author{
A. E. Cárcamo Hernández®, ${ }^{1, *}$ D. T. Huong $\oplus^{2, \dagger}$ and H. N. Long $\oplus^{3,4, \$}$ \\ ${ }^{1}$ Departamento de Física, Universidad Técnica Federico Santa María, \\ Casilla 110-V, Valparaíso 2340000, Chile \\ ${ }^{2}$ Institute of Physics, VAST, 10 Dao Tan, Ba Dinh, Hanoi 100000, Vietnam \\ ${ }^{3}$ Theoretical Particle Physics and Cosmology Research Group, Advanced Institute of Materials Science, \\ Ton Duc Thang University, Ho Chi Minh City 700000, Vietnam \\ ${ }^{4}$ Faculty of Applied Sciences, Ton Duc Thang University, Ho Chi Minh City 700000, Vietnam
}

(Received 31 October 2019; accepted 14 August 2020; published 8 September 2020)

\begin{abstract}
We propose a renormalizable theory with minimal particle content and symmetries, that successfully explains the number of Standard Model (SM) fermion families, the SM fermion mass hierarchy, the tiny values for the light active neutrino masses, the lepton and baryon asymmetry of the Universe, the dark matter relic density as well as the muon and electron anomalous magnetic moments. In the proposed model, the top quark and the exotic fermions do acquire tree-level masses whereas the SM charged fermions lighter than the top quark gain one-loop level masses. Besides that, the tiny masses for the light active neutrino are generated from an inverse seesaw mechanism at one-loop level.
\end{abstract}

DOI: $10.1103 /$ PhysRevD.102.055002

Despite the considerable experimental success of the Standard Model (SM), it leaves many unanswered questions such the origin of the observed SM fermion mass hierarchy, the number of SM fermion families, the tiny values of the light active neutrino masses, the nature of the dark matter (DM), the electric charge quantization, the lepton and baryon asymmetries of the Universe and the anomalous magnetic moments of the muon and electron. To address these issues, we propose a minimal renormalizable theory with the extended $S U(3)_{C} \times S U(3)_{L} \times U(1)_{X}$ gauge symmetry, which is supplemented by the $U(1)_{L_{g}}$ lepton number symmetry and the $Z_{4}$ discrete group. Its scalar and fermionic spectrum with their assignments under the symmetries of the model are shown in Tables I and II, respectively. The above gauge symmetry is crucial for explaining the number of SM fermion families since to fulfill the anomaly cancellation conditions, the number of left-handed $S U(3)_{L}$ fermion triplets has to be equal to the number of $S U(3)_{L}$ fermion antitriplets, which only happens when the number of fermion generations is a multiple of

\footnotetext{
*antonio.carcamo@usm.cl

†thuong@iop.vast.ac.vn

*hoangngoclong@tdtu.edu.vn
}

Published by the American Physical Society under the terms of the Creative Commons Attribution 4.0 International license. Further distribution of this work must maintain attribution to the author(s) and the published article's title, journal citation, and DOI. Funded by SCOAP ${ }^{3}$. three. Additionally, the charge quantization in theories with $S U(3)_{C} \times S U(3)_{L} \times U(1)_{X}$ gauge symmetry is explained thanks to the anomaly cancellation as shown for the first time in [1]. The $U(1)_{L_{g}}$ lepton number symmetry is spontaneously broken down to the residual $Z_{2}^{\left(L_{g}\right)}$ lepton number symmetry, under which only leptons are charged, thus forbidding proton decay [2]. In this model, the lepton number is defined as:

$$
L=\frac{4}{\sqrt{3}} T_{8}+L_{g},
$$

where $L_{g}$ is a conserved charge associated with the $U(1)_{L_{g}}$ global lepton number symmetry. Furthermore, the $Z_{4}$ discrete group is spontaneously broken down to the preserved $Z_{2}$ symmetry, which allows stable DM candidates that trigger a one-loop level radiative inverse seesaw mechanism for the generation of the light active neutrino masses. Such DM candidates also mediate the one-loop level radiative seesaw mechanism that produces the SM charged lepton masses.

Notice, as follows from Table I that the electrically neutral gauge singlet scalars $\eta$ and $\varrho$ as well as the neutral components of the $\phi$ scalar triplet are scalar DM candidates since their $Z_{4}$ charge corresponds to a nontrivial charge under the preserved $Z_{2}$ symmetry. In addition, from the fermionic assignments shown in Table II, it follows that $\Omega_{n R}(n=1,2)$ are fermionic DM candidates. The one-loop Feynman diagram contributing to the $\mu$ parameter of the 
TABLE I. Scalar assignments under $S U(3)_{C} \times S U(3)_{L} \times \times U(1)_{X} \times U(1)_{L_{q}} \times Z_{4}$.

\begin{tabular}{lcccccccccc}
\hline \hline & $\chi$ & $\rho$ & $\phi$ & $\sigma$ & $\varphi$ & $\varrho$ & $\eta$ & $S$ & $\zeta_{1}^{ \pm}$ & $\zeta_{2}^{ \pm}$ \\
\hline$S U(3)_{C}$ & $\mathbf{1}$ & $\mathbf{1}$ & $\mathbf{1}$ & $\mathbf{1}$ & $\mathbf{1}$ & $\mathbf{1}$ & $\mathbf{1}$ & 1 & $\mathbf{1}$ & $\mathbf{1}$ \\
$S U(3)_{L}$ & $\mathbf{3}$ & $\mathbf{3}$ & $\mathbf{3}$ & $\mathbf{1}$ & $\mathbf{1}$ & $\mathbf{1}$ & $\mathbf{1}$ & 1 & $\mathbf{1}$ & $\mathbf{1}$ \\
$U(1)_{X}$ & $-\frac{1}{3}$ & $\frac{2}{3}$ & $\frac{2}{3}$ & 0 & 0 & 0 & 0 & 0 & \pm 1 & \pm 1 \\
$U(1)_{L_{g}}$ & $\frac{4}{3}$ & $-\frac{2}{3}$ & $-\frac{2}{3}$ & 0 & 2 & 0 & 2 & 4 & 0 & 0 \\
$Z_{4}$ & 1 & 1 & $i$ & -1 & -1 & $-i$ & $-i$ & -1 & -1 & $-i$ \\
\hline \hline
\end{tabular}

TABLE II. Fermion assignments under $S U(3)_{C} \times S U(3)_{L} \times U(1)_{X} \times U(1)_{L_{q}} \times Z_{4}$. Here $n=1,2, k=1,3$ and $i=1,2,3$. Notice that there are six $S U(3)_{L}$ fermionic triplets (three from $Q_{1 L}$ and three from $L_{i L}$ ) and six $S U(3)_{L}$ fermionic antitriplets, i.e., $Q_{n L}$ $(n=1,2)$. In this counting the color quantum numbers are taken into account.

\begin{tabular}{lccccccccccccccccccccccc}
\hline \hline & $Q_{1 L}$ & $Q_{2 L}$ & $Q_{3 L}$ & $u_{i R}$ & $d_{i R}$ & $J_{1 R}$ & $J_{2 R}$ & $J_{3 R}$ & $T_{k L}$ & $T_{k R}$ & $T_{2 L}$ & $T_{2 R}$ & $B_{1 L}$ & $B_{1 R}$ & $B_{2 L}$ & $B_{2 R}$ & $L_{i L}$ & $l_{i R}$ & $E_{i L}$ & $E_{i R}$ & $N_{i R}$ & $\Omega_{n R}$ \\
\hline$S U(3)_{C}$ & $\mathbf{3}$ & $\mathbf{3}$ & $\mathbf{3}$ & $\mathbf{3}$ & $\mathbf{3}$ & $\mathbf{3}$ & $\mathbf{3}$ & $\mathbf{3}$ & $\mathbf{3}$ & $\mathbf{3}$ & $\mathbf{3}$ & $\mathbf{3}$ & $\mathbf{3}$ & $\mathbf{3}$ & $\mathbf{3}$ & $\mathbf{3}$ & $\mathbf{1}$ & $\mathbf{1}$ & $\mathbf{1}$ & $\mathbf{1}$ & $\mathbf{1}$ & $\mathbf{1}$ \\
$S U(3)_{L}$ & $\mathbf{3}$ & $\overline{\mathbf{3}}$ & $\overline{\mathbf{3}}$ & $\mathbf{1}$ & $\mathbf{1}$ & $\mathbf{1}$ & $\mathbf{1}$ & $\mathbf{1}$ & $\mathbf{1}$ & $\mathbf{1}$ & $\mathbf{1}$ & $\mathbf{1}$ & $\mathbf{1}$ & $\mathbf{1}$ & $\mathbf{1}$ & $\mathbf{1}$ & $\mathbf{3}$ & $\mathbf{1}$ & $\mathbf{1}$ & $\mathbf{1}$ & $\mathbf{1}$ & $\mathbf{1}$ \\
$U(1)_{X}$ & $\frac{1}{3}$ & 0 & 0 & $\frac{2}{3}$ & $-\frac{1}{3}$ & $\frac{2}{3}$ & $-\frac{1}{3}$ & $-\frac{1}{3}$ & $\frac{2}{3}$ & $\frac{2}{3}$ & $\frac{2}{3}$ & $\frac{2}{3}$ & $-\frac{1}{3}$ & $-\frac{1}{3}$ & $-\frac{1}{3}$ & $-\frac{1}{3}$ & $-\frac{1}{3}$ & -1 & -1 & -1 & 0 & 0 \\
$U(1)_{L_{g}}$ & $-\frac{2}{3}$ & $\frac{2}{3}$ & $\frac{2}{3}$ & 0 & 0 & -2 & 2 & 2 & 0 & 0 & 0 & 0 & 0 & 0 & 0 & 0 & $\frac{1}{3}$ & 1 & 1 & 1 & -1 & -1 \\
$Z_{4}$ & -1 & -1 & 1 & 1 & 1 & -1 & -1 & 1 & -1 & 1 & $-i$ & $-i$ & 1 & -1 & $i$ & $i$ & 1 & -1 & $i$ & $-i$ & 1 & $i$ \\
\hline \hline
\end{tabular}

inverse seesaw is shown in Fig. 1. Besides that, it is worth mentioning that the $Z_{4}$ discrete group allows preventing a tree-level charm quark mass, which in the model only appears at one-loop level. On the other hand, the appearance of tree-level up quark and SM down type quark masses is prevented due to the $U(1)_{X}$ assignments of the particle spectrum. Such masses are only generated at the one-loop level. The one-loop Feynman diagrams contributing to the entries of the SM charged fermion mass matrices are shown in Fig. 2. Notice that the electrically charged scalars together with heavy vectorlike up (down) type quarks mediate one-loop level radiative seesaw mechanisms that produce the masses for the down, strange and bottom quarks (up and charm quarks). It is worth mentioning that the set of heavy vectorlike quarks $T_{i}(i=1,2,3)$, $B_{n}(n=1,2)$ is the minimum amount of exotic quarks needed to provide one-loop level masses for the SM quarks

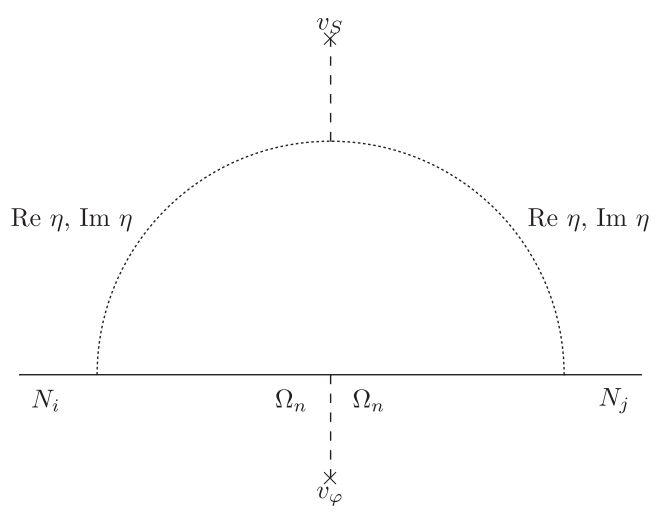

FIG. 1. One-loop Feynman diagram contributing to the Majorana neutrino mass submatrix $\mu$. Here, $n=1,2, i, j=1,2,3$. lighter than the top quark. In the case of minimal scalar content, one seesaw fermionic mediator is needed to provide one-loop level masses for each light SM fermions. Because of this reason, three vectors like-charged exotic
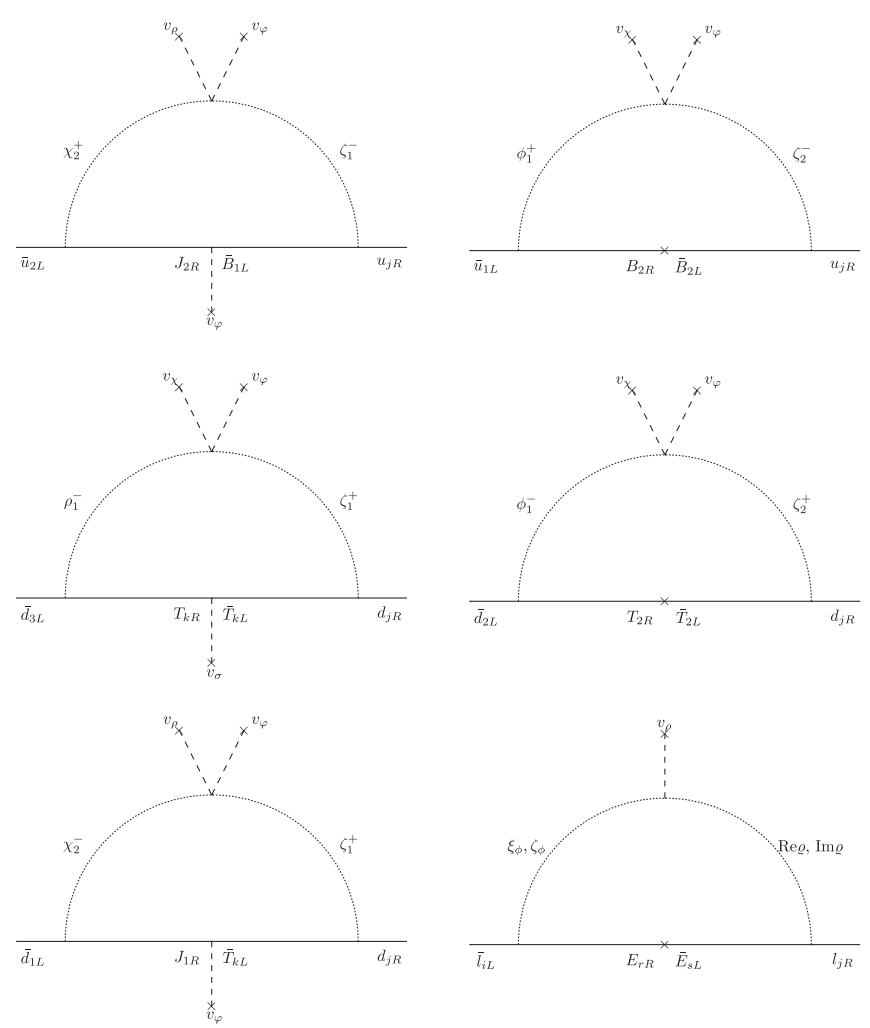

FIG. 2. One-loop Feynman diagrams contributing to the entries of the SM charged fermion mass matrices. Here, $n=1,2, k=1$, 3 and $i, j, r, s=1,2,3$. 
leptons $E_{i}(i=1,2,3)$ are needed to provide one-loop level masses for the SM charged leptons. Having several fermionic seesaw mediators lower than the number of SM fermions lighter than the top quark will yield a proportionality between rows and (or) columns of the SM fermion mass matrices yielding a vanishing determinant.

Furthermore, the $S U(3)_{L} \times U(1)_{X}$ gauge symmetry is also spontaneously broken down to the $S U(2)_{L} \times U(1)_{Y}$ symmetry, at the scale to the $\Lambda_{\text {int }}>\mathcal{O}(10) \mathrm{TeV}$, by the vacuum expectation value (VEV) of the third component of the $S U(3)_{L}$ scalar triplet $\chi$.

We consider $\Lambda_{\text {int }}>\mathcal{O}(10) \mathrm{TeV}$ to comply with collider [3] and flavor constraints [4-8]. The breaking of the $S U(2)_{L} \times U(1)_{Y}$ symmetry is triggered by the VEV of the second component of the $S U(3)_{L}$ scalar triplet $\rho$, whose $C P$ even neutral component corresponds to the $126 \mathrm{GeV}$ SM like Higgs boson. Consequently, the spontaneous symmetry breaking chain proceeds as follows:

$$
\begin{aligned}
\mathcal{G} & =S U(3)_{C} \times S U(3)_{L} \times U(1)_{X} \times U(1)_{L_{g}} \times Z_{4} \\
& \stackrel{\Lambda_{\text {int }}}{\rightarrow} S U(3)_{C} \times S U(2)_{L} \times U(1)_{Y} \times Z_{2}^{\left(L_{g}\right)} \times Z_{2} \\
& \stackrel{v_{\rho}}{\rightarrow} S U(3)_{C} \times U(1)_{Q} \times Z_{2}^{\left(L_{g}\right)} \times Z_{2} .
\end{aligned}
$$

The $S U(3)_{L}$ scalar triplets of this model are represented as:

$$
\begin{aligned}
& \chi=\left(\begin{array}{c}
\chi_{1}^{0} \\
\chi_{2}^{-} \\
\frac{1}{\sqrt{2}}\left(v_{\chi}+\xi_{\chi} \pm i \zeta_{\chi}\right)
\end{array}\right), \quad \phi=\left(\begin{array}{c}
\phi_{1}^{+} \\
\frac{1}{\sqrt{2}}\left(\xi_{\phi} \pm i \zeta_{\phi}\right) \\
\phi_{3}^{+}
\end{array}\right) \\
& \rho=\left(\begin{array}{c}
\rho_{1}^{+} \\
\frac{1}{\sqrt{2}}\left(v_{\rho}+\xi_{\rho} \pm i \zeta_{\rho}\right) \\
\rho_{3}^{+}
\end{array}\right)
\end{aligned}
$$

whereas the $S U(3)_{L}$ fermionic triplets and antitriplets take the form:

$$
\begin{aligned}
Q_{1 L} & =\left(u_{1}, d_{1}, J_{1},\right)_{L}^{T}, \quad Q_{n L}=\left(d_{n},-u_{n}, J_{n}\right)_{L}^{T}, \\
L_{i L} & =\left(\nu_{i}, l_{i}, \nu_{i}^{c}\right)_{L}^{T}, \quad n=2,3, \quad i=1,2,3 .
\end{aligned}
$$

where the first two components of the $S U(3)_{L}$ fermionic triplets and antitriplets correspond to the left-handed SM fermionic fields.

With the particle spectrum and symmetries specified in Tables I and II, we find that the relevant quark and lepton Yukawa interactions crucial to generate the observed SM fermion mass and mixing pattern, are described by the terms:

$$
\begin{aligned}
-\mathcal{L}_{Y}^{(q)}= & y_{1}^{(J)} \bar{Q}_{1 L} \chi J_{1 R}+\sum_{n=2}^{3} y_{n}^{(J)} \bar{Q}_{n L} \chi^{*} J_{n R}+m_{T} \bar{T}_{2 L} T_{2 R}+\sum_{k=1,3}\left(y_{k}^{(T)} \bar{T}_{k L} \sigma T_{k R}+x_{k}^{(J)} \bar{T}_{k L} \varphi J_{1 R}\right) \\
& +y_{B} \bar{B}_{1 L} \sigma B_{1 R}+m_{B} \bar{B}_{2 L} B_{2 R}+x_{J} \bar{B}_{1 L} \varphi^{*} J_{2 R}+\sum_{i=1}^{3} y_{i}^{(u)} \bar{Q}_{3 L} \rho^{*} u_{i R}+x_{2}^{(T)} \bar{Q}_{2 L} \phi^{*} T_{2 R} \\
& +\sum_{k=1,3}\left(x_{k}^{(T)} \bar{Q}_{3 L} \rho^{*} T_{k R}+\sum_{i=1}^{3} x_{k i}^{(d)} \bar{T}_{k L} \zeta_{1}^{+} d_{i R}\right)+\sum_{i=1}^{3} x_{2 i}^{(d)} \bar{T}_{2 L} \zeta_{2}^{+} d_{i R}+x_{B} \bar{Q}_{1 L} \phi B_{2 R} \\
& +\sum_{n=1}^{2} \sum_{i=1}^{3} z_{n i}^{(u)} \bar{B}_{n L} \zeta_{n}^{-} u_{i R}+\text { H.c., }
\end{aligned}
$$$$
-\mathcal{L}_{Y}^{(l)}=\sum_{i, j=1}^{3}\left[y_{i j}^{(N)} \bar{L}_{i L} \chi N_{j R}+x_{i j}^{(L)} \varepsilon_{a b c} \bar{L}_{i L}^{a}\left(L_{j L}^{C}\right)^{b}\left(\rho^{*}\right)^{c}\right]
$$$$
+\sum_{n=1}^{2}\left(\sum_{m=1}^{2} y_{n m}^{(\Omega)} \Omega_{n R} \overline{\Omega_{m R}^{C}} \varphi+\sum_{i=1}^{3} x_{\mathrm{in}}^{(N)} N_{i R} \overline{\Omega_{n R}^{C}} \eta\right)
$$$$
+\sum_{i, j=1}^{3}\left(x_{i j}^{(E)} \bar{L}_{i L} \phi E_{j R}+z_{i j}^{(l)} \bar{E}_{i L} \varrho l_{j R}\right)
$$$$
+\sum_{i=1}^{3}\left(m_{E}\right)_{i} \bar{E}_{i L} E_{i R}+\text { H.c. }
$$

After the model symmetries are spontaneously broken, the above Yukawa interactions generate the one-loop level

entries for the SM charged fermion mass matrices, as indicated by the Feynman diagrams of Fig. 2. In the SM quark sector, only the third row of the SM up type quark mass matrix is generated at tree level from the first term of the fourth line of Eq. (5). Such a third row is associated with the top quark mass which arises at tree level in the model. Furthermore, note that the terms between the first and third lines of Eq. (5) give rise to tree-level exotic quark masses. In addition, from the lepton Yukawa interactions of (6), it follows that the charged exotic leptons $E_{i}(i=1,2,3)$ (which are assumed to be physical fields) and the righthanded Majorana neutrinos $\Omega_{n R}(n=1,2)$ get tree-level masses at the scales $m_{E}$ and $v_{\varphi}$, respectively, whereas the SM charged leptons masses as well as the mass terms for the gauge singlet neutral leptons $N_{i R}(i=1,2,3)$ appear at 
one-loop level. Furthermore, from the quark Yukawa interactions, it follows that the exotic quarks $J_{i}(i=1$, $2,3), T_{2}, B_{2}$ and $B_{1}, T_{k}(k=1,3)$ do acquire treelevel masses at the scales $v_{\chi}, m_{T}, m_{B}$ and $v_{\sigma}$, respectively. Given that we are considering such scales to fulfill $\Lambda_{\text {int }}>\mathcal{O}(10) \mathrm{TeV}$, the exotic quark masses are larger than their LHC mass limit of $1.3 \mathrm{TeV}$ [9], for exotic Yukawa couplings of order unity. In addition, from the one loop Feynman diagrams of Fig. 2, we find that the SM quark mass matrices take the form:

$$
\begin{aligned}
& M_{U}=\left(\begin{array}{ccc}
\varepsilon_{11}^{(u)} & \varepsilon_{12}^{(u)} & \varepsilon_{13}^{(u)} \\
\varepsilon_{21}^{(u)} & \varepsilon_{22}^{(u)} & \varepsilon_{23}^{(u)} \\
y_{1}^{(u)} & y_{2}^{(u)} & y_{3}^{(u)}
\end{array}\right) \frac{v}{\sqrt{2}}, \quad i=1,2,3, \\
& \varepsilon_{1 i}^{(u)}=\frac{1}{16 \pi^{2}} \frac{\lambda_{\phi^{\dagger} \chi \varphi^{*} \zeta_{2}^{+}} x_{B} z_{2 i}^{(u)} v_{\chi} v_{\varphi}}{m_{B_{2}} v} C_{0}\left(\frac{m_{\phi_{1}^{+}}}{m_{B_{2}}}, \frac{m_{\zeta_{2}^{-}}}{m_{B_{2}}}\right) \\
& =\frac{1}{16 \pi^{2}} \frac{x_{B} z_{2 i}^{(u)} A_{1}}{m_{B_{2}}} C_{0}\left(\frac{m_{\phi_{1}^{+}}}{m_{B_{2}}}, \frac{m_{\zeta_{2}^{-}}}{m_{B_{2}}}\right) \\
& \varepsilon_{2 i}^{(u)}=\frac{1}{16 \pi^{2}} \frac{\lambda_{\rho^{\dagger} \chi \varphi^{*} \zeta_{1}^{+}} y_{2}^{(J)} z_{1 i}^{(u)} v_{\varphi}}{m_{J_{2} B_{1}}} C_{0}\left(\frac{m_{\chi_{2}^{+}}}{m_{J_{2} B_{1}}}, \frac{m_{\zeta_{1}^{-}}}{m_{J_{2} B_{1}}}\right) \text {, } \\
& =\frac{1}{16 \pi^{2}} \frac{y_{2}^{(J)} z_{1 i}^{(u)} A_{2}}{m_{J_{2} B_{1}}} C_{0}\left(\frac{m_{\chi_{2}^{+}}}{m_{J_{2} B_{1}}}, \frac{m_{\zeta_{1}^{-}}}{m_{J_{2} B_{1}}}\right) \\
& M_{D}=\left(\begin{array}{ccc}
\varepsilon_{11}^{(d)} & \varepsilon_{12}^{(d)} & \varepsilon_{13}^{(d)} \\
\varepsilon_{21}^{(d)} & \varepsilon_{22}^{(d)} & \varepsilon_{23}^{(d)} \\
\varepsilon_{31}^{(d)} & \varepsilon_{32}^{(d)} & \varepsilon_{33}^{(d)}
\end{array}\right) \frac{v}{\sqrt{2}}, \quad i=1,2,3, \\
& \varepsilon_{1 i}^{(d)}=\frac{1}{16 \pi^{2}} \sum_{k=1,3} \frac{\lambda_{\rho \chi^{\dagger} \varphi \zeta_{1}^{-}} y_{1}^{(J)} x_{k i}^{(d)} v_{\varphi}}{m_{J_{1} T_{k}}} C_{0}\left(\frac{m_{\chi_{2}^{-}}}{m_{J_{1} T_{k}}}, \frac{m_{\zeta_{1}^{+}}}{m_{J_{1} T_{k}}}\right) \text {, } \\
& =\sum_{k=1,3} \frac{y_{1}^{(J)} x_{k i}^{(d)} A_{2}}{16 \pi^{2} m_{J_{1} T_{k}}} C_{0}\left(\frac{m_{\chi_{2}^{-}}}{m_{J_{1} T_{k}}}, \frac{m_{\zeta_{1}^{+}}}{m_{J_{1} T_{k}}}\right) \\
& \varepsilon_{2 i}^{(d)}=\frac{1}{16 \pi^{2}} \frac{\lambda_{\phi \chi^{\dagger} \varphi \zeta_{2}^{-}} x_{2}^{(T)} x_{2 i}^{(d)} v_{\chi} v_{\varphi}}{m_{T_{2}} v} C_{0}\left(\frac{m_{\phi_{1}^{-}}}{m_{T_{2}}}, \frac{m_{\zeta_{2}^{+}}}{m_{T_{2}}}\right) \text {, } \\
& =\frac{x_{2}^{(T)} x_{2 i}^{(d)} A_{1}}{16 \pi^{2} m_{T_{2}}} C_{0}\left(\frac{m_{\phi_{1}^{-}}}{m_{T_{2}}}, \frac{m_{\zeta_{2}^{+}}}{m_{T_{2}}}\right) \\
& \varepsilon_{3 i}^{(d)}=\frac{1}{16 \pi^{2}} \sum_{k=1,3} \frac{\lambda_{\rho \chi^{\dagger} \varphi \zeta_{2}^{-}} x_{k}^{(T)} x_{k i}^{(d)} v_{\chi} v_{\varphi}}{m_{T_{k}} v} C_{0}\left(\frac{m_{\rho_{1}^{-}}}{m_{T_{k}}}, \frac{m_{\zeta_{1}^{+}}}{m_{T_{k}}}\right) \\
& =\sum_{k=1,3} \frac{x_{k}^{(T)} x_{k i}^{(d)} A_{3}}{16 \pi^{2} m_{T_{k}}} C_{0}\left(\frac{m_{\rho_{1}^{-}}}{m_{T_{k}}}, \frac{m_{\zeta_{1}^{+}}}{m_{T_{k}}}\right)
\end{aligned}
$$

where the following loop function has been introduced [10]:
$C_{0}\left(\hat{m}_{1}, \hat{m}_{2}\right)=\frac{\hat{m}_{1}^{2} \hat{m}_{2}^{2} \ln \left(\frac{\hat{m}_{1}^{2}}{\hat{m}_{2}^{2}}\right)-\hat{m}_{1}^{2} \ln \hat{m}_{1}^{2}+\hat{m}_{2}^{2} \ln \hat{m}_{2}^{2}}{\left(1-\hat{m}_{1}^{2}\right)\left(1-\hat{m}_{2}^{2}\right)\left(\hat{m}_{1}^{2}-\hat{m}_{2}^{2}\right)}$.

The experimental values of the SM quark masses and Cabbibo-Kobayashi-Maskawa (CKM) parameters [11-13]:

$$
\begin{aligned}
m_{u}(\mathrm{MeV}) & =1.45_{-0.45}^{+0.56}, \quad m_{d}(\mathrm{MeV})=2.9_{-0.4}^{+0.5} \\
m_{s}(\mathrm{MeV}) & =57.7_{-15.7}^{+16.8}, \quad m_{c}(\mathrm{MeV})=635 \pm 86 \\
m_{t}(\mathrm{GeV}) & =172.1 \pm 0.6 \pm 0.9, \quad m_{b}(\mathrm{GeV})=2.82_{-0.04}^{+0.09} \\
\sin \theta_{12} & =0.2254, \sin \theta_{23}=0.0414, \quad \sin \theta_{13}=0.00355 \\
J & =2.96_{-0.16}^{+0.20} \times 10^{-5},
\end{aligned}
$$

can be well reproduced for the following benchmark point:

$$
\begin{aligned}
z_{11}^{(u)} & \simeq 0.1, \quad z_{12}^{(u)} \simeq 2.39, \quad z_{13}^{(u)} \simeq 0.1, \\
z_{21}^{(u)} & \simeq 0.44, \quad z_{22}^{(u)} \simeq 0.1, \quad z_{23}^{(u)} \simeq 0.1, \\
x_{11}^{(d)} & \simeq 0.69+1.53 i, \quad x_{12}^{(d)} \simeq 0.025-0.86 i, \\
x_{21}^{(d)} & \simeq-1.12-3.46 i, \quad x_{22}^{(d)} \simeq 0.18+3.53 i, \\
x_{23}^{(d)} & \simeq 2.25-0.59 i, \quad y_{1}^{(u)} \simeq-0.65, \quad x_{13}^{(d)} \simeq 0.85 \\
x_{31}^{(d)} & \simeq-1.74-3.49 i, \quad x_{32}^{(d)} \simeq 0.08+3.43 i, \\
x_{1}^{(T)} & \simeq 0.95, \quad x_{2}^{(T)} \simeq-1.3, \quad x_{3}^{(T)} \simeq 1.25, \\
y_{1}^{(J)} & \simeq 0.12, \quad y_{2}^{(J)} \simeq 1.54, \quad x_{B} \simeq 0.57 \\
A_{2} & \simeq 1.9 \mathrm{TeV}, \quad A_{3} \simeq 4 \mathrm{TeV}, \quad m_{B_{2}} \simeq 7.7 \mathrm{TeV}, \\
m_{J_{2} B_{1}} & \simeq 7.7 \mathrm{TeV}, \quad m_{T_{1}} \simeq 26 \mathrm{TeV}, \quad m_{T_{2}} \simeq 6 \mathrm{TeV}, \\
m_{J T_{1}} & \simeq 928 \mathrm{GeV}, \quad m_{J T_{3}} \simeq 500 \mathrm{GeV}, \\
m_{\rho_{1}^{ \pm}} & \simeq 100 \mathrm{GeV}, \quad x_{33}^{(d)} \simeq 0.51, \quad y_{3}^{(u)} \simeq-0.44, \\
m_{\zeta_{1}^{ \pm}} & \simeq 5 \mathrm{TeV}, \quad m_{\zeta_{2}^{ \pm}} \simeq 2.7 \mathrm{TeV}, \quad m_{\chi_{2}^{ \pm}} \simeq 5 \mathrm{TeV}, \\
m_{\phi_{1}^{ \pm}} & \simeq 5.5 \mathrm{TeV}, \quad m_{T_{3}} \simeq 32 \mathrm{TeV}, \quad y_{2}^{(u)} \simeq-0.61 .
\end{aligned}
$$

Note that we use the experimental values of the quark masses at the $M_{Z}$ scale, from Ref. [12], which are similar to those in [11]. The experimental values of the CKM parameters are taken from Ref. [13]. Furthermore, from the neutrino Yukawa interactions of Eq. (6) we get the following neutrino mass terms:

$$
-\mathcal{L}_{\text {mass }}^{(\nu)}=\frac{1}{2}\left(\begin{array}{lll}
\overline{\nu_{L}^{C}} & \overline{\nu_{R}} & \overline{N_{R}}
\end{array}\right) M_{\nu}\left(\begin{array}{c}
\nu_{L} \\
\nu_{R}^{C} \\
N_{R}^{C}
\end{array}\right)+\text { H.c. }
$$

where the full neutrino mass matrix is given by: 


$$
M_{\nu}=\left(\begin{array}{ccc}
0_{3 \times 3} & \frac{v_{\rho}}{2 \sqrt{2}} z^{(L)} & 0_{3 \times 3} \\
\frac{v_{\rho}}{2 \sqrt{2}}\left(z^{(L)}\right)^{T} & 0_{3 \times 3} & \frac{v_{\chi}}{\sqrt{2}} y^{(N)} \\
0_{3 \times 3} & \frac{v_{\chi}}{\sqrt{2}}\left(y^{(N)}\right)^{T} & \mu
\end{array}\right)
$$

with

$$
z^{(L)}=\left(x^{(L)}\right)^{\dagger}-\left(x^{(L)}\right)^{*}
$$

and the entries of the submatrix, which are generated at one-loop level from the Feynman diagram of Fig. 1, take the form [14]:

$$
\begin{aligned}
\mu_{i j}= & \sum_{n=1}^{2} \frac{x_{\mathrm{in}}^{(N)} x_{j n}^{(N)}\left(m_{\Omega}\right)_{n m}}{16 \pi^{2}}\left[\frac{m_{\eta_{R}}^{2}}{m_{\eta_{R}}^{2}-\left(m_{\Omega}\right)_{n m}^{2}} \ln \left(\frac{m_{\eta_{R}}^{2}}{\left(m_{\Omega}\right)_{n m}^{2}}\right)\right. \\
& \left.-\frac{m_{\eta_{I}}^{2}}{m_{\eta_{I}}^{2}-\left(m_{\Omega}\right)_{n m}^{2}} \ln \left(\frac{m_{\eta_{I}}^{2}}{\left(m_{\Omega}\right)_{n m}^{2}}\right)\right]
\end{aligned}
$$

where $i, j=1,2,3,\left(m_{\Omega}\right)_{n m}=y_{n m}^{(\Omega)} v_{\varphi}(n, m=1,2)$, $m_{\eta_{R}}=m_{R e \eta}, m_{\eta_{I}}=m_{I m \eta}$.

We would like to note that the neutrinos $\nu_{a L}, \nu_{a R}$ carry the lepton number one unit and the fields $N_{a R}$ have an opposite lepton number with respect to that of neutrinos. The Majorana mass term of $N_{a R}, \mu$-parameter, does not conserve the lepton number. The smallness of $\mu$ can be naturally realized via a radiative correction given in (14). For another one-loop level realization of the inverse seesaw mechanism see for instance [15].

Diagonalization of the neutrino mass matrix (13) for $\mu, \frac{v_{\rho}}{2 \sqrt{2}} z^{(L)} \ll \frac{v_{\chi}}{\sqrt{2}} y^{(N)}$ leads to the physical neutrino mass matrices as follows

$$
\begin{aligned}
& M_{\nu}^{(1)} \simeq\left(\frac{v_{\rho}}{v_{\chi}}\right)^{2} z^{(L)}\left(\left(y^{(N)}\right)^{T}\right)^{-1} \mu\left(y^{(N)}\right)^{-1}\left(z^{(L)}\right)^{T}, \\
& M_{\nu}^{(2)} \simeq \frac{v_{\chi}}{2 \sqrt{2}}\left(y^{(N)}+\left(y^{(N)}\right)^{T}\right)-\frac{1}{2} \mu, \\
& M_{\nu}^{(3)} \simeq \frac{v_{\chi}}{2 \sqrt{2}}\left(y^{(N)}+\left(y^{(N)}\right)^{T}\right)+\frac{1}{2} \mu,
\end{aligned}
$$

where $M_{\nu}^{(1)}$ corresponds to the mass matrix for light active neutrinos $\left(\nu_{a}\right)$, whereas $M_{\nu}^{(2)}$ and $M_{\nu}^{(3)}$ are the mass matrices for sterile neutrinos $\left(N_{a}^{-}, N_{a}^{+}\right)$which are superpositions of mostly $\nu_{a R}$ and $N_{a R}$ as $N_{a}^{ \pm} \sim \frac{1}{\sqrt{2}}\left(\nu_{a R} \mp N_{a R}\right)$. In the limit $\mu \rightarrow 0$, which corresponds to unbroken lepton number, we recover the massless neutrinos of the SM. Due to the smallness of the $\mu$ - parameter, the mass splitting of three pairs of sterile neutrinos becomes small, thus implying that the sterile neutrinos form pseudo-Dirac pairs and the light active neutrino mass scale resulting from the inverse seesaw mechanism takes the form: [16]

$$
\left(\frac{M_{\nu}^{(1)}}{0.1 \mathrm{eV}}\right)=\left(\frac{\frac{v_{\rho}}{2 \sqrt{2}} z_{L}^{(N)}}{100 \mathrm{GeV}}\right)^{2}\left(\frac{\mu}{1 \mathrm{keV}}\right)\left(\frac{M_{\nu}^{(2,3)}}{10^{4} \mathrm{GeV}}\right)^{-2}
$$

Considering the neutrino Yukawa couplings, $x^{(N)}$, of order unity, assuming for simplicity degenerate Majorana neutrinos $\Omega_{n R}(n=1,2)$ with a common mass of $20 \mathrm{TeV}$, $m_{\eta_{R}}=400 \mathrm{GeV}$, with the splitting $m_{\eta_{R}}-m_{\eta_{I}} \simeq 0.63 \mathrm{MeV}$, one sees that $\mu \simeq 1 \mathrm{keV}$. Thus, the light neutrino mass can be $0.1 \mathrm{eV}$ if heavy neutrino mass scale is a few ten $\mathrm{TeV}$ or less and the Dirac neutrino mass is order of $100 \mathrm{GeV}$. This means that this model can account for the smallness of the light active neutrino masses and several singlet pseudoDirac fermions have $O(1) \mathrm{TeV}$ masses. As mentioned above, the pseudo-Dirac fermions are degenerate in mass and thus they allow the implementation of the resonant leptogenesis mechanism crucial to generate the baryon asymmetry of the universe (BAU) [17]. In the inverse seesaw mechanism, the smallness of the $\mu$-parameter can naturally suppress the washout of pre-existing baryon asymmetry [18,19]. However, to explain the smallness of the $\mu$-parameter, the model introduces exotic fermions and scalar fields. They create new washout processes as

$$
\begin{aligned}
& l H^{+} \rightarrow N_{a R} \backslash \nu_{a R} \rightarrow \eta \Omega_{a R},\left(E_{j R}, \phi_{3}^{+}\right), \\
& l H^{+} \rightarrow N_{a R} \rightarrow \chi_{1}^{0} \bar{\nu}_{a},
\end{aligned}
$$

where $H^{ \pm} \sim \rho_{1}^{ \pm} \cos \alpha+\chi_{2}^{ \pm} \sin \alpha$. In the case, the baryogenesis temperature $\left(T_{B G}\right)$ higher than the inverse seesaw scale $\Lambda_{I S S}$, the washout processes can only be avoided if the Yukawa couplings related to the processes given in Eq. (17) must be too tiny. So the radiative inverse seesaw mechanism is insignificant. The singlets $\Omega_{a R}$ generate the small lepton number violation $\mu$-parameter via one-loop correction [see in Eq. (14)], the baryogenesis occurs before electroweak symmetry breaking. In the case $\Lambda_{\mathrm{EM}}<T_{B G}<$ $\Lambda_{I S S}$, if at least one particle has a mass lower than the value of the baryogenesis temperature, the washout process can be suppressed [20]. We assume that $y^{(N)}$ and $x^{(N)}$ are diagonal matrices and that there is an inverse hierarchical structure between them as $y_{33}^{(N)} \ll y_{22}^{(N)}, y_{11}^{(N)}$. This choice allows us to successfully accommodate the experimental value of the electron and muon anomalous magnetic moments as indicated by Eq. (33). Therefore only the third generation of $N_{a}^{ \pm}$can give the contribution to the BAU. We also assume that the masses of $E_{a R}, \Omega_{a R}$ fields are larger than the masses of the lightest pseudo-Dirac fermions $N_{3}^{ \pm} \equiv N^{ \pm}$, the lepton asymmetry parameter, which is induced by decay process of $N^{ \pm}$, has the following form $[21,22]$ 


$$
\begin{aligned}
\epsilon_{ \pm} & =\frac{\sum_{\alpha=1}^{3}\left\{\Gamma\left(N_{ \pm} \rightarrow l_{\alpha} H^{+}\right)-\Gamma\left(N_{ \pm} \rightarrow \bar{l}_{\alpha} H^{-}\right)\right\}}{\sum_{\alpha=1}^{3}\left\{\Gamma\left(N_{ \pm} \rightarrow l_{\alpha} H^{+}\right)+\Gamma\left(N_{ \pm} \rightarrow \bar{l}_{\alpha} H^{-}\right)\right\}} \\
& \simeq \frac{\operatorname{Im}\left(h_{\nu}^{\dagger} h_{N} h_{\nu}^{\dagger} h_{N}\right)_{33}}{8 \pi A_{ \pm}} \frac{r}{r^{2}+\frac{\Gamma_{\mp}^{2}}{m_{N^{\mp}}^{2}}}
\end{aligned}
$$

where we have defined $h_{\nu}=-y^{(N)} \sin \alpha+z^{(L)} \cos \alpha, h_{N}=$ $y^{(N)} \sin \alpha+z^{(L)} \cos \alpha$, and

$$
\begin{aligned}
r & \equiv \frac{m_{N^{+}}^{2}-m_{N^{-}}^{2}}{m_{N^{+}} m_{N^{-}}} \simeq \frac{2 \mu}{\frac{v_{x}}{2 \sqrt{2}}\left(y^{N}+\left(y^{N}\right)^{T}\right)}, \\
A_{-} & =\left(h_{N}^{\dagger} h_{N}\right)_{33}, \quad A_{+}=\left(h_{\nu}^{\dagger} h_{\nu}\right)_{33}, \quad \Gamma_{ \pm}=\frac{A_{ \pm} m_{N_{1}^{ \pm}}}{8 \pi} .
\end{aligned}
$$

If one neglects the interference terms involving the two different sterile neutrinos $N^{ \pm}$, the washout parameter $K_{N^{+}}+K_{N^{-}}$is huge as mentioned in [23]. However, the small mass splitting between the pseudo-Dirac neutrinos leads to a destructive interference in the scattering process [18]. The washout parameter including the interference term is given as follows

$$
K^{\mathrm{eff}} \simeq\left(K_{N^{+}}+K_{N^{-}}\right) \delta^{2}
$$

with $\delta=\frac{m_{N^{+}}-m_{N^{-}}}{\Gamma_{N^{ \pm}}}, K_{N^{ \pm}}=\frac{\Gamma_{ \pm}}{H}$ and $H(T)=\sqrt{\frac{4 \pi^{3} g^{*}}{45} \frac{T^{2}}{M_{P}}}$, where $g^{*}=118$ is the number of effective relativistic degrees of freedom and $M_{\mathrm{Pl}}=1.2 \times 10^{9} \mathrm{GeV}$ is the Planck constant. In order to estimate the effective washout parameter, we use the extended Casas-Ibarra parametrization for the case of the inverse seesaw mechanism [23]

$$
z^{(L)}=\frac{2 v_{\chi}}{v_{\rho}}\left(U_{P M N S} M_{\nu}^{\frac{1}{2}} R \mu^{\frac{-1}{2}} y^{(N)}\right),
$$

where, $U_{P M N S}$ is the Pontecorvo-Maki-Nakagawa-Sakata (PMNS) leptonic mixing mass matrix, whose Standard parametrization is given by:

$$
\left(\begin{array}{ccc}
c_{12} c_{13} & s_{12} c_{13} & s_{13} e^{-i \delta} \\
-s_{12} c_{23}-c_{12} s_{23} s_{13} e^{i \delta} & c_{12} c_{23}-s_{12} s_{23} s_{13} e^{-i \delta} & s_{23} c_{13} \\
s_{12} s_{23}-c_{12} c_{23} s_{13} e^{i \delta} & -c_{12} s_{23}-s_{12} c_{23} s_{13} e^{i \delta} & c_{23} c_{13}
\end{array}\right)
$$

with $c_{i j}=\cos \theta_{i j}, s_{i j}=\sin \theta_{i j}$. Notice that, for the sake of simplicity, we dropped the Majorana phases in Eq. (22).

For normal ordering, the current best fit values for $\theta_{i j}$ and the $C P$ violating phase are determined by [24]

$$
\begin{array}{ll}
\frac{s_{12}^{2}}{10^{-1}}=3.20_{-0.16}^{+0.20}, & \frac{s_{23}^{2}}{10^{-1}}=5.47_{-0.30}^{+0.20}, \\
\frac{s_{13}^{2}}{10^{-2}}=2.160_{-0.069}^{+0.083} ; & \frac{\delta}{\pi}=1.21_{-0.15}^{+0.21} .
\end{array}
$$

Furthermore, $M_{\nu}=\operatorname{diag}\left(m_{\nu_{1}}, m_{\nu_{2}}, m_{\nu_{3}}\right)$, being $m_{\nu_{i}}(i=1$, $2,3)$ the light active neutrino masses. The Dirac neutrino mass matrix is antisymmetric, thus implying that one light active neutrino is massless. Then, the light active neutrino masses for the case of normal hierarchy are given by:

$$
m_{1}=0, \quad m_{2}=\sqrt{\left|\Delta m_{21}^{2}\right|}, \quad m_{3}=\sqrt{\left|\Delta m_{31}^{2}\right|},
$$

where the neutrino mass squared differences have the following experimental values [24]:

$$
\begin{aligned}
& \Delta m_{21}^{2}\left[10^{-5} \mathrm{eV}^{2}\right]=7.55_{-0.16}^{+0.20}, \\
& \Delta m_{31}^{2}\left[10^{-3} \mathrm{eV}^{2}\right]=2.50 \pm 0.03 .
\end{aligned}
$$

Besides that $R$ is a complex orthogonal matrix which can be parametrized by

$R=\left(\begin{array}{ccc}c_{y} c_{z} & -s_{x} c_{z} s_{y}-c_{x} s_{z} & s_{x} s_{z}-c_{x} s_{y} c_{z} \\ c_{y} s_{z} & c_{x} c_{z}-s_{x} s_{y} s_{z} & -c_{z} s_{x}-c_{x} s_{y} s_{z} \\ s_{y} & s_{x} c_{y} & c_{x} c_{y}\end{array}\right)$,

where $c_{x}=\cos x, s_{x}=\sin x$ and so on, being $x, y, z \in C$. For simplicity, we assume $x=y=z=\theta=\mathcal{R}[\theta]+i \mathcal{I}[\theta]$ and we work on the basis where $y^{(N)}=\operatorname{Diag}\left(y_{1}^{(N)}\right.$, $\left.y_{2}^{(N)}, y_{3}^{(N)}\right)$ is a diagonal matrix. In the Fig. 3, we plot the washout parameter as a function of $\mathcal{I}[\theta]$ for $\mu=1 \mathrm{keV}, v_{\chi}=10^{5} \mathrm{GeV}, v_{\rho}=246 \mathrm{GeV}$, and $\mathcal{R}[\theta]=$ $1.2 \pi, y_{11}^{(N)} \simeq 0.9, y_{22}^{(N)} \simeq 0.5 i$ and $y_{3}^{(N)}=0.01$.

The washout parameter depends on the imaginary part of complex angle $\theta$ and the mixing angle $\alpha$ (see in Fig.(3)). For $\alpha=\frac{\pi}{2}$, we predict an extremely high value of the washout parameter: $K_{\text {eff }} \simeq 10^{3}$.

For $\alpha \neq \frac{\pi}{2}$, the model predicts both weak and strong washout regions. Especially, the $\theta$ angle is real, the washout parameter is larger than unit for any value of $\alpha$. In the weak and strong washout region, the baryon asymmetry is related to the lepton asymmetry [22] as follows

$\eta_{b}=\frac{n_{B}}{s}=-\frac{28}{79} \frac{\epsilon_{ \pm}}{g^{*}}, \quad$ for $K^{\text {eff }} \ll 1$,

$\eta_{b}=\frac{n_{B}}{s}=-\frac{28}{79} \frac{0.3 \epsilon_{ \pm}}{g^{*} K^{\mathrm{eff}}\left(\ln K^{\mathrm{eff}}\right)^{0.6}}, \quad$ for $K^{\mathrm{eff}} \gg 1$,

Figure 4 shows the baryon asymmetry $\eta_{b}$ as a function of $\mathcal{I}[\theta]$ for the difference choice $\alpha$ in the strong washout region. The amount of baryon asymmetry can reach its experimental value $\eta_{B}=6.2 \times 10^{-10}$. The value of $\mathcal{I}[\theta]$ 


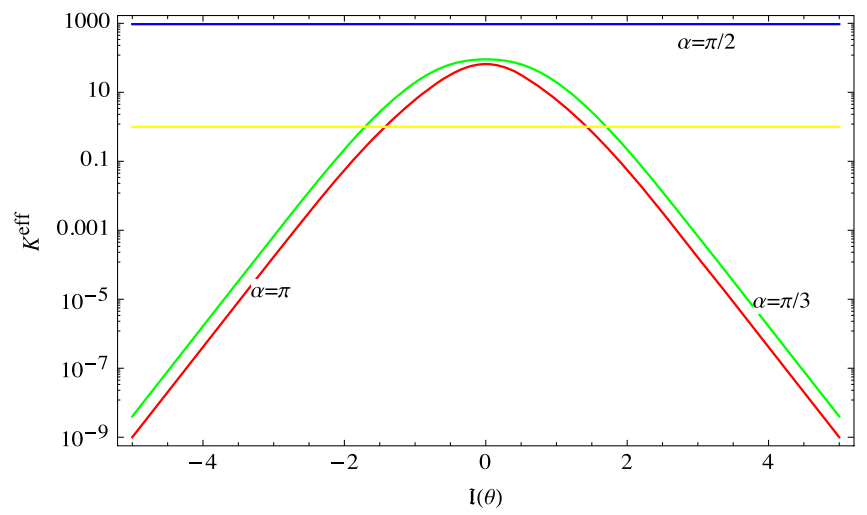

FIG. 3. Effective washout parameter as a function of $\mathcal{I}[\theta]$ for different values of $\alpha$.

that allows solving the baryon asymmetry depends on the value of the mixing angle $\alpha$. From results shown in the Figs. 4, we can realize:

(i) The amount of baryon asymmetry strongly depends on the mixing angle $\alpha$.

(ii) If $\theta$ is a real number, the predicted results are consistent with the results given in [23].

As $\alpha$ approaches $\frac{\pi}{2}$, one gets into the strong washout regime, the amount of baryon asymmetry suppressed. Figure 5 displays the amount of baryon asymmetry in the weak washout region via using a logarithmic scale for numerical data over a range of $\alpha$ value. At $\alpha=\frac{\pi}{2}$ or $\alpha=\frac{3 \pi}{2}$, the curves are pulled because the suppression of the lepton asymmetry

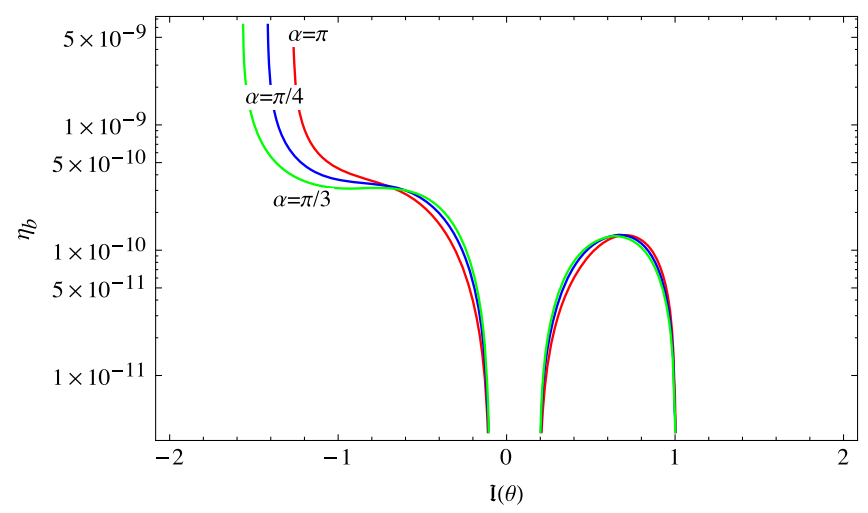

FIG. 4. Baryon asymmetry as a function of $\mathcal{I}[\theta]$ for fixing the mixing angle $\alpha$ in the strong washout region.

parameter. From the results shown in Fig. 5, one can find values of $\mathcal{I}[\theta]$ and $\alpha$ that allows creating a sufficient amount of baryon asymmetry consistent with its experimental value.

On the other hand, given that we are considering the exotic charged leptons $E_{1}, E_{2}$ and $E_{3}$ as physical fields, the SM charged lepton mass matrix is diagonal and the lepton mixings only arise from the neutrino sector. Consequently, to reproduce the experimental values of the neutrino mass squared splittings, leptonic mixing angles, and the leptonic Dirac $C P$-violating phase, for the scenario of normal neutrino mass hierarchy, we obtain the following numerical solution for the light active neutrino mass matrix:

$$
M_{\nu}^{(1)}=\left(\begin{array}{ccc}
0.00298903-0.00104607 i & -0.00131313+0.00347814 i & -0.00661584+0.00316521 i \\
-0.00131313+0.00347814 i & 0.0299126+0.000395314 i & 0.0213737-5.363173174949189 \times 10^{-6} i \\
-0.00661584+0.00316521 i & 0.0213737-5.363173174949189 \times 10^{-6} i & 0.0249309-0.000337142 i
\end{array}\right) \mathrm{eV}
$$

It is worth mentioning that such light active neutrino mass matrix generated from the one-loop level inverse seesaw mechanism allows to generate enough baryon asymmetry. Furthermore, from the light active neutrino mass matrix given above, we obtain that the effective Majorana neutrino mass parameter of neutrinoless double beta decay takes the value of around $3.2 \mathrm{meV}$, which is beyond the reach of the present and forthcoming $0 \nu \beta \beta$ decay experiments.

It is worth mentioning that the chosen benchmark of diagonal SM charged lepton mass matrix as well as diagonal $y^{(N)}$ matrix allows to have very suppressed charged lepton flavor violating (CLFV) processes. Note that the CLFV process $\mu \rightarrow e \gamma$ receives one loop-level contributions arising from vertex diagrams involving the exchange of heavy $W^{\prime}$ and the heavy sterile neutrinos. Given that we are considering the case of diagonal $y^{(N)}$

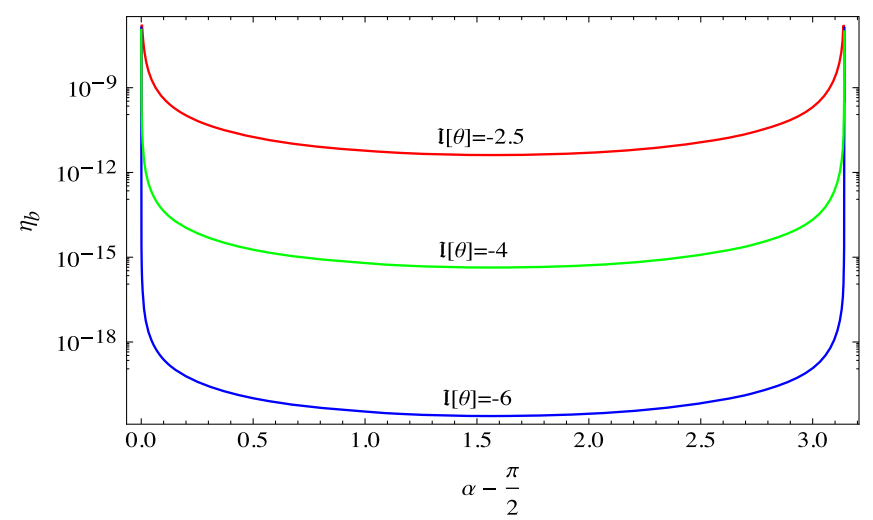

FIG. 5. Baryon asymmetry as a function of the mixing angle $\alpha$ in the weak washout region. 
matrix, the off-diagonal entries of the sterile neutrino mass matrices will be very small compared with the diagonal ones, as follows from Eq. (15), thus implying that the $\mu \rightarrow e \gamma$ will be suppressed by the square of the off-diagonal entries of the sterile neutrino mass matrices. Thus, our model fulfill the constraints arising from CLFV processes.

On the other hand, as previously mentioned, the model has several DM candidates. Here we assume that the lightest of them is the gauge singlet scalar $\eta_{I}$. Such singlet scalar dark matter candidate $\eta_{I}$ mainly annihilates into $W W, Z Z, t \bar{t}, b \bar{b}$ and $H H$ via the Higgs portal quartic scalar interaction $H^{2} \eta_{I}^{2}$ that arises from the $\lambda_{\eta \rho} \eta \eta^{\dagger} \rho \rho^{\dagger}$ term of the scalar potential. We have numerically checked that in order to successfully reproduce the experimental value, $\Omega h^{2}=0.1198 \pm 0.0026$ [25], of the relic density, the mass $m_{\eta_{I}}$ of the scalar field $\eta_{I}$ has to be in the range $400 \mathrm{GeV} \lesssim m_{\eta_{I}} \lesssim 800 \mathrm{GeV}$, for a quartic scalar coupling $\lambda_{\eta \rho}$ in the range $1 \lesssim \lambda_{\eta \rho} \lesssim 1.5$. Notice that we have taken $m_{\eta_{I}} \simeq m_{\eta_{R}}=400 \mathrm{GeV}$, which corresponds to the lower bound for the mass of the singlet scalar DM candidate, as shown in Ref [26], where the scalar DM candidate is also a gauge singlet as in our model. This bound is consistent with the current constraints arising from LUX and PandaX-II experiments [27,28]. In what concerns DM-direct-detection prospects, the scalar DM candidate would scatter off a nuclear target in a detector via Higgs boson exchange in the $t$-channel, thus yielding a constraint on the coupling of the $\left(\rho^{\dagger} \rho\right) \eta_{I} \eta_{I}$ interaction. The corresponding scattering cross section takes the form [29]:

$$
\sigma_{D M-p, n} \simeq 5 \times 10^{-44} \lambda_{\eta \rho}^{2}\left(\frac{1 \mathrm{TeV}}{m_{\mathrm{DM}}}\right)^{2} \mathrm{~cm}^{2}
$$

Using the experimental value of the dark matter relic density, we find that the scattering cross section is predicted around $7 \times 10^{-45} \mathrm{~cm}^{2}$, which is consistent with direct detection limits from the LUX [30] and XENON1T [31] experiments. In addition, note that the Yukawa terms $x_{n}^{(E)} \bar{L}_{n L} \phi E_{n R}$ and $z_{n}^{(l)} \bar{E}_{n L} \varrho l_{n R}(n=1,2)$, as well as the trilinear scalar interaction $A \rho \phi^{\dagger} Q^{*}$, will give rise to oneloop level contributions to the muon and electron anomalous magnetic moments. Such one-loop level contributions to the $\left(g_{e, \mu}-2\right)$ will involve the exchange of $C P$-even and $C P$-odd scalars as well as of the heavy charged exotic leptons $E_{n}(n=1,2)$ running in the internal lines of the triangular loops. Besides that, the muon and electron anomalous magnetic moments also receive one loop-level contributions arising from vertex diagrams involving the exchange of the electrically charged scalar $\chi_{2}^{+}$, which couples to the right-handed Majorana neutrinos $N_{s R}$ ( $s=1$ for electron and $s=2$ for muon). Then, the electron and muon anomalous magnetic moments take the form:

$$
\begin{aligned}
\Delta a_{e, \mu}= & \frac{y_{1,2}^{2} m_{e, \mu}^{2}}{8 \pi^{2}}\left[I_{S}^{(1,2)}\left(m_{E_{1,2}}, m_{H_{1}}\right)-I_{S}^{(1,2)}\left(m_{E_{1,2}}, m_{H_{2}}\right)\right. \\
& \left.+I_{P}^{(1,2)}\left(m_{E_{1,2}}, m_{A_{1}}\right)-I_{P}^{(1,2)}\left(m_{E_{1,2}}, m_{A_{2}}\right)\right] \sin \theta \cos \theta \\
& -\frac{\left(y_{11,22}^{(N)}\right)^{2} m_{e, \mu}^{2}}{8 \pi^{2} m_{\chi_{2}^{ \pm}}^{2}} J\left(\frac{m_{\mu_{11,22}}}{m_{e, \mu}}, \frac{m_{\mu_{11,22}}}{m_{\chi_{2}^{ \pm}}}\right)
\end{aligned}
$$

where $H_{1}=\cos \theta_{S} \operatorname{Re} \varrho+\sin \theta_{S} \xi_{\phi}, H_{2}=-\sin \theta_{S} \operatorname{Re} \varrho+$ $\cos \theta_{S} \xi_{\phi}, A_{1}=\cos \theta_{P} \operatorname{Im} \varrho+\sin \theta_{P} \xi_{\phi}, A_{2}=-\sin \theta_{P} \operatorname{Im} \varrho+$ $\cos \theta_{P} \zeta_{\phi}$ and for the sake of simplicity we have set $x_{n}^{(E)}=$ $z_{n}^{(l)}=y_{n}(n=1,2), \theta_{S}=\theta_{P}$. In addition, the loop integrals are given by [32-34]:

$$
\begin{aligned}
& I_{S(P)}^{(1,2)}\left(m_{E}, m\right) \\
& \quad=\int_{0}^{1} \frac{x^{2}\left(1-x \pm \frac{m_{E}}{m_{e, \mu}}\right) d x}{m_{e, \mu}^{2} x^{2}+\left(m_{E}^{2}-m_{e, \mu}^{2}\right) x+m^{2}(1-x)}, \\
& J\left(\frac{m_{\mu_{11,22}}}{m_{e, \mu}}, \frac{m_{\mu_{11,22}}}{m_{\chi_{2}^{ \pm}}}\right) \\
& \quad=\int_{0}^{1} d x \frac{P_{+}\left(x, \frac{m_{\mu_{11,22}}}{m_{e, \mu}}\right)+P_{-}\left(x, \frac{m_{\mu_{11,22}}}{m_{e, \mu}}\right)}{\left(\frac{m_{\mu_{11,22}}}{m_{\chi_{2}^{ \pm}}}\right)^{2}(1-x)\left[1-\left(\frac{m_{e, \mu}}{m_{\mu_{11,22}}}\right)^{2} x\right]+x}
\end{aligned}
$$

with

$$
P_{ \pm}(x, \epsilon)=-x(1-x)(x \pm \epsilon)
$$

Notice that whereas the charged exotic vectorlike lepton $E_{1}$ contributes to the electron anomalous magnetic moment, $E_{2}$ contributes to the muon anomalous magnetic moment. This is due to the fact that we are considering the exotic charged leptons $E_{1}$ and $E_{2}$ as well as $E_{3}$ as physical fields, thus implying a diagonal SM charged lepton mass matrix, where the SM charged lepton masses are given by:

$m_{i}=\frac{y_{i}^{2}}{2}\left[F\left(m_{H_{1}}, m_{A_{1}}, m_{E_{i}}\right)-F\left(m_{H_{2}}, m_{A_{2}}, m_{E_{i}}\right)\right] \sin 2 \theta$

being $i=1,2,3$ and the loop function given by [14]:

$$
\begin{aligned}
F\left(m_{1}, m_{2}, m_{3}\right)= & \frac{m_{3}}{16 \pi^{2}}\left[\frac{m_{1}^{2}}{m_{1}^{2}-m_{3}^{2}} \ln \left(\frac{m_{1}^{2}}{m_{3}^{2}}\right)\right. \\
& \left.-\frac{m_{2}^{2}}{m_{2}^{2}-m_{3}^{2}} \ln \left(\frac{m_{2}^{2}}{m_{3}^{2}}\right)\right],
\end{aligned}
$$

From the previous relations we find that the SM charged lepton masses [35] and the muon anomalous magnetic moment can be very well reproduced for the following benchmark point: 


$$
y_{1} \simeq 0.43, \quad y_{2} \simeq 1.01, \quad y_{3} \simeq 3.07,2 \mathrm{em} \theta=\frac{\pi}{4},
$$

$m_{E_{1}} \simeq 5.6 \times 10^{5} \mathrm{TeV}, \quad m_{E_{2}} \simeq 1.8 \times 10^{2} \mathrm{TeV}$,

$m_{E_{3}} \simeq 2.7 \times 10^{3} \mathrm{TeV}, \quad m_{H_{1}} \simeq 10 \mathrm{TeV}, \quad m_{H_{2}} \simeq 5.2 \mathrm{TeV}$,

$m_{A_{1}} \simeq 18 \mathrm{TeV}, \quad m_{A_{2}} \simeq 14.5 \mathrm{TeV}, \quad m_{\chi_{2}^{ \pm}} \simeq 0.1 \mathrm{TeV}$,

$y_{11}^{(N)} \simeq 1.1, \quad y_{22}^{(N)} \simeq 0.7 i, \quad\left|\mu_{11}\right|=\left|\mu_{22}\right| \simeq 0.1 \mathrm{keV}$.

Then, for such benchmark point we get the following values for the muon and electron anomalous magnetic moments:

$$
\Delta a_{\mu} \simeq 22.5 \times 10^{-10}, \quad \Delta a_{e} \simeq-1.6 \times 10^{-13} .
$$

This shows that the obtained values for the muon and electron anomalous magnetic moments are consistent within the $1 \sigma$ and $2 \sigma$ experimentally allowed ranges, respectively, with their corresponding experimental values [36-39]:

$$
\begin{aligned}
& \left(\Delta a_{\mu}\right)_{\exp }=(26.1 \pm 8) \times 10^{-10}, \\
& \left(\Delta a_{e}\right)_{\exp }=(-0.88 \pm 0.36) \times 10^{-12} .
\end{aligned}
$$

In conclusion, we have constructed a minimal renormalizable theory that successfully explains the number of SM fermion generations, the electric charge quantization, the SM fermion mass hierarchy, the tiny values of the light active neutrino masses, the lepton and baryon asymmetry of the Universe, the observed DM relic density as well as the muon and electron anomalous magnetic moments. In our model, the top quark, as well as the exotic fermions, obtain tree-level masses, whereas the SM charged fermions lighter than the top quark get one-loop level masses. Besides that, the tiny light active neutrino masses are produced from a one-loop level inverse seesaw mechanism.

\section{ACKNOWLEDGEMENTS}

This research has received funding from Fondecyt (Chile), Grants No. 1170803; ICP. No. 2020.02; the Vietnam National Foundation for Science and Technology Development (NAFOSTED) under Grant No. 103.01-2019.312. A. E. C.H is very grateful to the Institute of Physics, VAST for the warm hospitality and for financing his visit.
[1] P. V. Dong and H. N. Long, Int. J. Mod. Phys. A 21, 6677 (2006).

[2] A. E. Cárcamo Hernández, S. Kovalenko, H. N. Long, and I. Schmidt, J. High Energy Phys. 07 (2018) 144.

[3] C. Salazar, R. H. Benavides, W. A. Ponce, and E. Rojas, J. High Energy Phys. 07 (2015) 096.

[4] A. E. Carcamo Hernandez, R. Martinez, and F. Ochoa, Phys. Rev. D 73, 035007 (2006).

[5] R. Martinez and F. Ochoa, Phys. Rev. D 77, 065012 (2008).

[6] A. J. Buras, F. De Fazio, and J. Girrbach, J. High Energy Phys. 02 (2014) 112.

[7] A. J. Buras, F. De Fazio, and J. Girrbach-Noe, J. High Energy Phys. 08 (2014) 039.

[8] A. J. Buras, F. De Fazio, J. Girrbach, and M. V. Carlucci, J. High Energy Phys. 02 (2013) 023.

[9] M. Aaboud et al. (ATLAS Collaboration), Phys. Rev. Lett. 121, 211801 (2018).

[10] C. Arbeláez, A. E. Cárcamo Hernández, S. Kovalenko, and I. Schmidt, Eur. Phys. J. C 77, 422 (2017).

[11] Z. z. Xing, H. Zhang, and S. Zhou, Phys. Rev. D 77, 113016 (2008).

[12] K. Bora, Horizons in biochemistry and biophysics 2, 112 (2013).

[13] M. Tanabashi et al. (Particle Data Group), Phys. Rev. D 98, 030001 (2018).

[14] E. Ma, Phys. Rev. D 73, 077301 (2006).
[15] P. S. B. Dev and A. Pilaftsis, Phys. Rev. D 86, 113001 (2012).

[16] F. Deppisch and J. W. F. Valle, Phys. Rev. D 72, 036001 (2005).

[17] A. Pilaftsis and T.E. J. Underwood, Phys. Rev. D 72, 113001 (2005).

[18] S. Blanchet, T. Hambye, and F. X. Josse-Michaux, J. High Energy Phys. 04 (2010) 023.

[19] S. Blanchet, P. S. B. Dev, and R. N. Mohapatra, Phys. Rev. D 82, 115025 (2010).

[20] I. Baldes, N. F. Bell, K. Petraki, and R. R. Volkas, J. Cosmol. Astropart. Phys. 07 (2013) 029.

[21] P. H. Gu and U. Sarkar, Phys. Lett. B 694, 226 (2010).

[22] A. Pilaftsis, Phys. Rev. D 56, 5431 (1997).

[23] M. J. Dolan, T. P. Dutka, and R. R. Volkas, J. Cosmol. Astropart. Phys. 06 (2018) 012.

[24] P. F. de Salas, D. V. Forero, C. A. Ternes, M. Tortola, and J. W. F. Valle, Phys. Lett. B 782, 633 (2018).

[25] P. A. R. Ade et al. (Planck Collaboration), Astron. Astrophys. 594, A13 (2016).

[26] N. Bernal, A. E. Cárcamo Hernández, I. de Medeiros Varzielas, and S. Kovalenko, J. High Energy Phys. 05 (2018) 053.

[27] D. S. Akerib et al. (LUX Collaboration), Phys. Rev. Lett. 118, 021303 (2017).

[28] A. Tan et al. (PandaX-II Collaboration), Phys. Rev. Lett. 117, 121303 (2016). 
[29] C. Garcia-Cely and A. Ibarra, Nucl. Part. Phys. Proc. 263-264, 107 (2015).

[30] D. S. Akerib et al. (LUX Collaboration), Nucl. Instrum. Methods Phys. Res., Sect. A 704, 111 (2013).

[31] E. Aprile (XENON1T Collaboration), Springer Proc. Phys. 148, 93 (2013).

[32] E. Ma and M. Raidal, Phys. Rev. Lett. 87, 011802 (2001); 87, 159901(E) (2001).

[33] R. A. Diaz, R. Martinez, and J. A. Rodriguez, Phys. Rev. D 67, 075011 (2003).
[34] C. Kelso, H. N. Long, R. Martinez, and F. S. Queiroz, Phys. Rev. D 90, 113011 (2014).

[35] Z. Z. Xing, Phys. Rep. 854, 1 (2020).

[36] K. Hagiwara, R. Liao, A. D. Martin, D. Nomura, and T. Teubner, J. Phys. G 38, 085003 (2011).

[37] T. Nomura and H. Okada, Phys. Dark Universe 26, 100359 (2019).

[38] T. Nomura and H. Okada, Phys. Rev. D 97, 095023 (2018).

[39] R. H. Parker, C. Yu, W. Zhong, B. Estey, and H. Müller, Science 360, 191 (2018). 